\title{
Muscle pathology helps predict JDM outcomes
}

The use of
longitudinal
clinical data
collected in
the JDCBS
enabled
modelling of
the disease
course over
time

In juvenile dermatomyositis (JDM) prognostic biomarkers are lacking, but longitudinal modelling of treatment status using data from the UK JDM Cohort and Biomarker Study (JDCBS) now suggests that muscle pathology and myositis-specific autoantibody (MSA) status could be used to predict long-term outcomes. "In our prognostic model, we found that muscle biopsy score can predict a patient's probability of remaining on medication over time [and] interestingly, the effect of muscle biopsy score [is] influenced by the patients' autoantibody status," reports Claire Deakin, who led the analysis.

Deakin et al. found that muscle pathology, analysed using a standardized scoring tool, correlated with MSA subtype such that patients with certain MSAs (particularly anti-Mi2 antibodies) had high biopsy scores, reflecting severe pathology, whereas other MSAs (such as antiMDA5 antibodies) were associated with low biopsy scores, indicating mild pathology. "Although it was previously known that certain clinical features of JDM correlated with MSA subtypes, any correlations between muscle pathology and MSA subtypes were unknown before this study," recalls Deakin.

The use of longitudinal clinical data collected in the JDCBS enabled modelling of the disease course over time. For patients with anti-TIF1 $\gamma$ or anti-NXP2 autoantibodies, or no detectable autoantibodies, a high biopsy score was associated with an increased probability of remaining on medication. Interestingly, however, patients with anti-Mi2 autoantibodies had a lower probability of remaining on treatment despite having more severe muscle pathology at biopsy; by contrast, those with anti-MDA5 autoantibodies, who had mild muscle pathology, seemed more likely to remain on medication over time.

"To take this work forward, it will be important to validate these findings in an independent cohort of [patients with JDM]," explains Deakin, highlighting that such work will be challenging to complete given the need to collect a large number of biopsy samples and longitudinal clinical data, which would require international collaboration. "We are also keen to investigate biological mechanisms underpinning MSA subtypes, including genetic variants and dysregulated pathways," Deakin continues. "In particular, we would like to understand why some patients with certain MSAs, such as anti-Mi2 [antibodies], fare better than others in their clinical course."

Sarah Onuora

ORIGINAL ARTICLE Deakin, C. T. et al. Muscle biopsy in combination with myositis-specific autoantibodies aids prediction of outcomes in juvenile dermatomyositis. Arthritis Rheumatol. http://dx.doi.org/10.1002/art.39753 (2016) 